dc-switchable and single-nanocrystal-addressable coherent population transfer

Deniz Gunceler, and Ceyhun Bulutay

Citation: Appl. Phys. Lett. 97, 241909 (2010);

View online: https://doi.org/10.1063/1.3526751

View Table of Contents: http://aip.scitation.org/toc/apl/97/24

Published by the American Institute of Physics

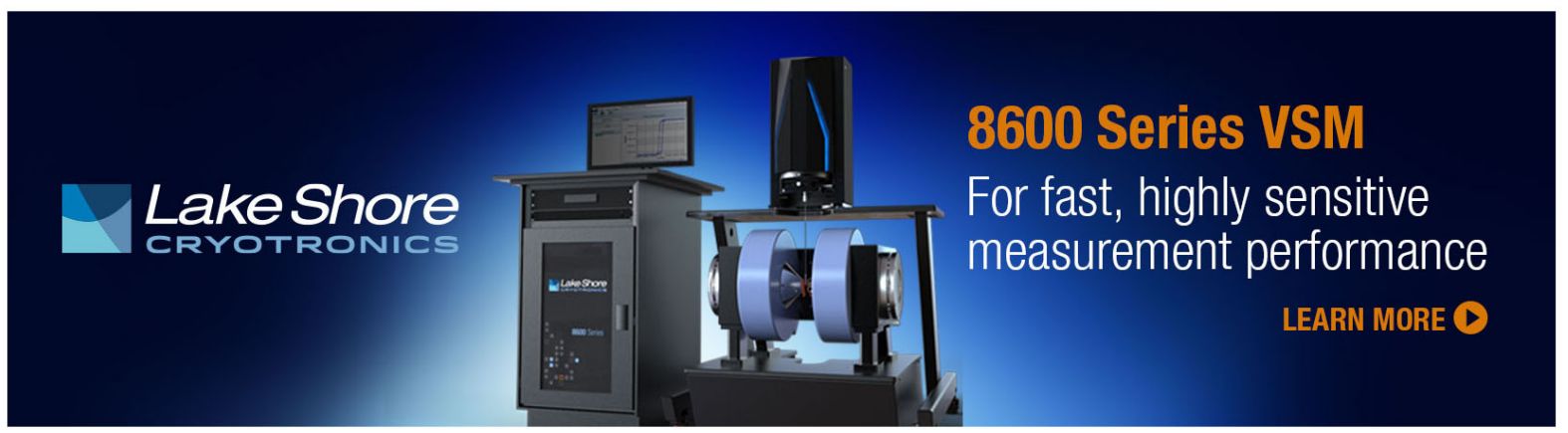




\title{
dc-switchable and single-nanocrystal-addressable coherent population transfer
}

\author{
Deniz Gunceler ${ }^{\mathrm{a})}$ and Ceyhun Bulutay ${ }^{\mathrm{b})}$ \\ Department of Physics, Bilkent University, 06800 Bilkent, Ankara, Turkey
}

(Received 8 September 2010; accepted 18 November 2010; published online 13 December 2010)

Achieving coherent population transfer in the solid-state is challenging compared to atomic systems
due to closely spaced electronic states and fast decoherence. Here, within an atomistic
pseudopotential theory, we offer recipes for the stimulated Raman adiabatic passage in embedded
silicon and germanium nanocrystals. The transfer efficiency spectra display characteristic Fano
resonances. By exploiting the Stark effect, we predict that transfer can be switched off with a dc
voltage. As the population transfer is highly sensitive to structural variations, with a choice of a
sufficiently small two-photon detuning bandwidth, it can be harnessed for addressing individual
nanocrystals within an ensemble. @ 2010 American Institute of Physics. [doi:10.1063/1.3526751]

The control of the dynamics of quantum systems using coherent optical beams lies at the heart of quantum information technologies. ${ }^{1,2}$ Among several alternatives, the stimulated Raman adiabatic passage (STIRAP) offers a certain degree of robustness in atomic systems with respect to laser parameter fluctuations. ${ }^{3}$ Its solid-state implementation was recently achieved in $\operatorname{Pr}^{+3}: \mathrm{Y}_{2} \mathrm{SiO}_{5}$ crystal, ${ }^{4,5}$ and $\mathrm{Tm}^{+3}: \mathrm{YAG}$ (yttrium aluminum garnet) crystal, ${ }^{6}$ all at cryogenic temperatures. Next obvious milestone is to demonstrate STIRAP in nanocrystals (NCs) embedded in a host lattice. Compared to rare-earth doped ions in inorganic solids, ${ }^{4-6}$ NCs bring further flexibility in the design of the functional units by tailoring the physical parameters such as material composition, size, shape, and strain, together with the external fields. However, the challenge with NCs is that the charge degrees of freedom are more susceptible to decoherence compared to the atomic or "impurity" systems. 7,8

In this work we consider silicon and germanium NCs embedded in silica. Our aim here is to explore from a theoretical standpoint the feasibility as well as the intricacies of STIRAP in this system. To set the stage, first we need to address the constraints imposed by decoherence on our system. The ultimate decoherence mechanism is the radiative recombination. The typical radiative lifetimes of $\mathrm{Si}$ and $\mathrm{Ge}$ $\mathrm{NCs}$ are in the microsecond range whereas for direct band gap semiconductors this is in the nanosecond range. ${ }^{9}$ Another recombination channel, in case multiple electrons get excited by a strong laser pulse, is the Auger process. According to our recent theoretical estimation for the excited-electron configuration of Auger recombination in $\mathrm{Si}$ and $\mathrm{Ge} \mathrm{NCs}$, this lifetime is in the range of subnanoseconds. ${ }^{10}$ An even more critical decoherence channel in NCs is the acoustic phonon scattering. ${ }^{7,8}$ For the case of InGaAs quantum dots, Borri et al. ${ }^{11}$ demonstrated close to radiative limit linewidth at $7 \mathrm{~K}$, corresponding to a dephasing time of $630 \mathrm{ps}$. In Si NCs, Sychugov et al. ${ }^{12}$ showed that the linewidth can also be as sharp as direct band gap materials, reaching $2 \mathrm{meV}$ at 35 K. A similar system is the excited Rydberg states ${ }^{2}$ of phosphorus-doped silicon having a spatial extent of $\sim 10 \mathrm{~nm}$ for which a dephasing time of $\sim 320$ ps is very recently

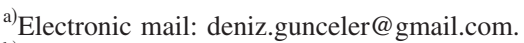

${ }^{b)}$ Electronic mail: bulutay@bilkent.edu.tr.
}

predicted. ${ }^{13}$ Guided by these reports, we aim for a complete STIRAP in less than 300 ps so that at sufficiently low temperatures of a few Kelvins this can beat the decoherence clock in Si and Ge NCs. Admittedly, this is a cautiously optimistic estimate; nevertheless a worse case can still be accommodated by further scaling the pulse widths and laser powers accordingly; thanks to high-field tolerance of silica embedded NCs.

Our theoretical model involves the atomistic description of the system within a supercell geometry of several thousand atoms most of which are the surrounding matrix atoms. Initially, nearly spherical $\mathrm{NCs}$ in $C_{3 v}$ point symmetry are considered, and in the final part consequences of shape deformation are discussed. The local potential is represented as a superposition of screened semiempirical pseudopotentials of the constituent atoms; ${ }^{14}$ the spin-orbit interaction is particularly included, since this coupling among closely spaced levels can potentially affect the selection rules and hence the transfer efficiency. A dc electric field is also accounted nonperturbatively for the Stark field analysis. The excitonic effects are ignored as the confinement energy dominates for small NCs. ${ }^{8}$ To solve the single-particle Schrödinger equation for such a large number of atoms with sufficient accuracy up to the highly excited states, we make use of the linear combination of bulk bands approach. ${ }^{15}$

The population transfer is built on this atomistic electronic structure, as shown in the insets of Fig. 1. The electric dipole coupling is used for the interaction with the pump and Stokes beams. Unlike atomic systems, in the case of NCs, we have to consider multiple intermediate ${ }^{16,17}$ and final states. On the other hand we assume a single initial state, namely, the highest occupied molecular orbital (HOMO). However, by selecting the interaction parameters accordingly (see Table I), we have assured that the maximum probability of finding a sub-HOMO electron in the conduction band is quite negligible. Our computations show that had the interaction individually involved any such sub-HOMO electron as the initial state, its transfer probability to the conduction band would have an upper bound of $10^{-9} \%$. Finally, since the intermediate state is not populated for the case of an ideal STIRAP, we neglect many-body effects due to Pauli blocking. 

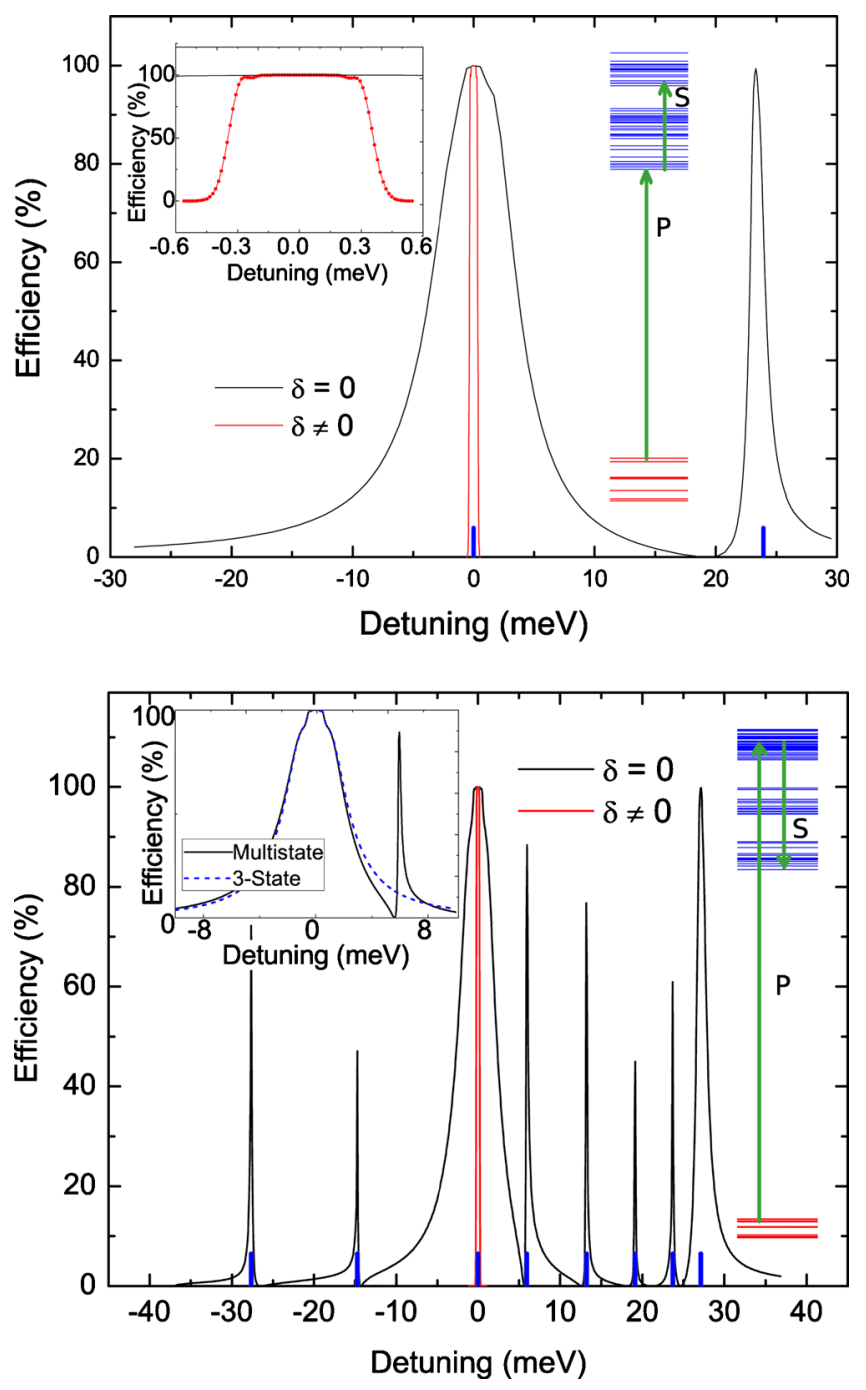

FIG. 1. (Color online) The population transfer efficiency as the pump laser is detuned for the $1.5 \mathrm{~nm} \mathrm{Ge} \mathrm{NC} \mathrm{(top)} \mathrm{and} \mathrm{the} 2.1 \mathrm{~nm} \mathrm{Si} \mathrm{NC} \mathrm{(bottom),} \mathrm{with}$ $(\delta=0)$ and without two-photon resonance $(\delta \neq 0)$. The left inset in the upper graph is a close-up for the central peak. The short blue lines on the detuning axis the energies of the intermediate states. The insets on the right show the electronic states and laser energies.

We base our discussions on a spherical $2.1 \mathrm{~nm}$ diameter $\mathrm{Si} \mathrm{NC}$ and a $1.5 \mathrm{~nm}$ Ge NC, purposely selected because of their close band gaps of 2.74 and $2.80 \mathrm{eV}$, respectively. Considering the oscillator strength of the transitions, we adopt different schemes for the two NCs (see right insets in Fig. 1). For a $1.5 \mathrm{~nm}$ Ge NC the HOMO to lowest unoccupied mo-

TABLE I. Laser parameters optimized for STIRAP for the $2.1 \mathrm{~nm} \mathrm{Si}$, and $1.5 \mathrm{~nm}$ Ge NCs. The incident electric fields are specified for free-space medium. Delay refers to time between the peaks of the Stokes and pump pulses both with Gaussian profiles.

\begin{tabular}{llccc}
\hline \hline & $\begin{array}{c}\text { E-field } \\
(\mathrm{MV} / \mathrm{cm})\end{array}$ & $\begin{array}{c}\text { Wavelength } \\
(\mathrm{nm})\end{array}$ & $\begin{array}{c}\text { FWHM } \\
(\mathrm{ps})\end{array}$ & $\begin{array}{c}\text { Delay } \\
(\mathrm{ps})\end{array}$ \\
\hline Pump & 1.5569 & $\mathrm{Si} \mathrm{NC}$ & & \\
Stokes & 0.058954 & 336.9 & 90 & 75 \\
& & 1323 & & \\
Pump & 0.32325 & Ge NC & & \\
Stokes & 0.92810 & 148.4 & 60 & 50 \\
\hline \hline
\end{tabular}

lecular orbital (LUMO) transition is quite strong; therefore, we utilize the ladder scheme where the electron is transferred from the HOMO state to the $\mathrm{LUMO}+28$ state $(0.837 \mathrm{eV}$

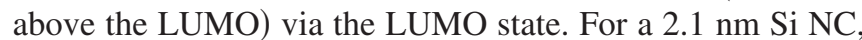
we utilize a $\Lambda$ scheme where the electron is transferred from the HOMO state to the LUMO state via the LUMO+30 state (0.937 eV above the LUMO). For the pump and Stokes pulses, we use counterintuitively time-ordered Gaussian profiles ${ }^{3}$ giving rise to equal peak Rabi frequencies with values 0.35 and $0.55 \mathrm{THz}$ for $\mathrm{Si}$ and Ge NCs, respectively. Both pulses are linearly polarized but in certain directions chosen to optimize the transfer. We refer to Table I for the other laser parameters.

The transfer efficiency is plotted in Fig. 1. In all the figures, detuning refers to that of the pump pulse; in the case of two-photon resonance, the Stokes pulse is assumed to be detuned by the same amount from its targeted transition. In the other case, the Stokes pulse is kept resonant. The first observation we make is that the transfer efficiency is superior when the two-photon resonance condition is satisfied. This fact is well documented in literature. ${ }^{3}$ When the system has two-photon detuning, the central peak shrinks down into a plateau, where the transfer efficiency is essentially constant, and suddenly drops to zero (see upper inset).

Another noteworthy aspect of Fig. 1 is the presence of side peaks. The detuning values for these peaks coincide with the resonance condition restored with a neighboring intermediate state, marked by the blue vertical bars in Fig. 1. As the laser parameters were optimized for the central peak and not for the neighbors, these peaks are usually not as tall or wide. Also since there are no states immediately below the LUMO (ladder scheme), for the $1.5 \mathrm{~nm}$ Ge NC there are no such peaks for negative detuning. The peaks display the asymmetrical well-known Fano lineshape. ${ }^{18,19}$ In a similar context, this was observed in the tunneling-induced transparency in quantum well intersubband transitions. ${ }^{20}$ It arises from two paths interfering with opposite phase on one of the two sides of the resonance. This is illustrated in the lower left inset of Fig. 1: For small detunings the calculations made without considering any neighbors agree very well with the full calculation. However, as soon as there is enough detuning to transfer the electron through one of the neighboring states, the neighbors-removed treatment cannot reproduce the dip right before the second peak in the solid line that occurs due to the interference between the chosen intermediate state and its neighbor.

Next, in Fig. 2, we investigate the effect of an external dc electric field (see the inset). Note that we quote the matrix dc electric fields, viz., outside the $\mathrm{NC}$ in the embedding re-

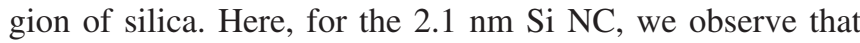
for small fields, the transfer efficiency is not affected, but the intermediate population pile-up increases. After a critical field of $0.35 \mathrm{MV} / \mathrm{cm}$, transfer efficiency rapidly drops to zero. To identify its origin, we first assured that the system is robust against changes in the dipole matrix elements; hence Rabi frequencies are not significantly altered by the dc field. On the other hand, NC energy levels undergo significant Stark shifts, the valence states being more so compared to conduction states, as revealed by our recent work. ${ }^{21} \mathrm{We}$ checked that in this field range it does not give rise to a level crossing between the HOMO and the lower-lying states. Hence, the primary mechanism responsible for this switching is the Stark shift-induced two-photon detuning, something 


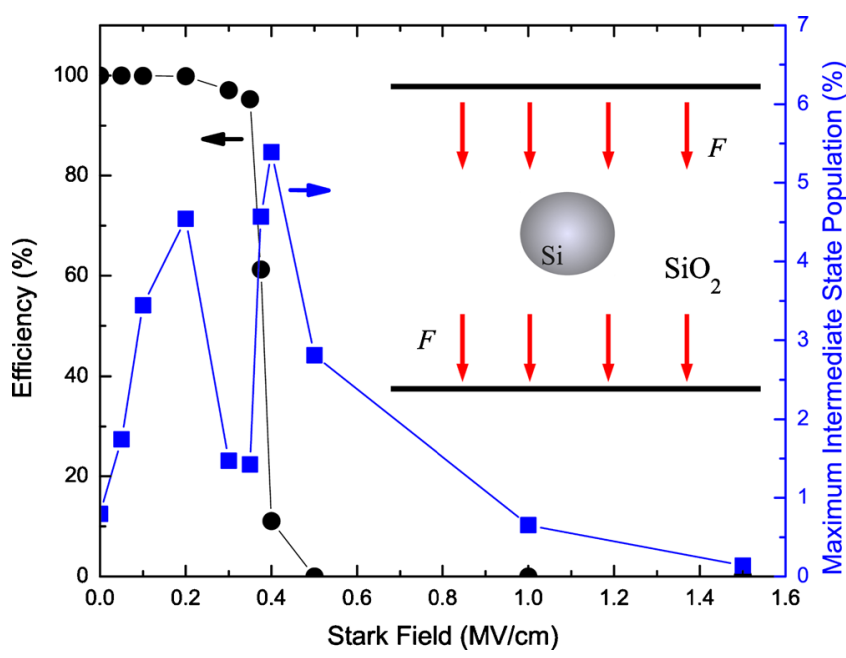

FIG. 2. (Color online) Effect of dc Stark field on the overall transfer efficiency and maximum intermediate-state population for the $2.1 \mathrm{~nm} \mathrm{Si} \mathrm{NC}$. The lines are only to guide the eyes.

STIRAP is very sensitive to. For the zero-field case, a detuning of $0.3 \mathrm{meV}$ is enough to destroy STIRAP. This value of Stark shift is reached at $0.5 \mathrm{MV} / \mathrm{cm}$, after which the population transfer is quenched.

The highly critical two-photon bandwidth is controllable by the time delay between Stokes and pump pulses, which is illustrated in Fig. 3. As the overlap between these pulses is reduced the two-photon bandwidth first increases up to a delay of $80 \mathrm{ps}$, beyond which it retracts back, as expected from the fundamental principles of STIRAP that demands a nonzero overlap between the two pulses. ${ }^{3}$ The upper inset shows the buildup of the intermediate-state population away from the two-photon resonance.

Finally, we focus on the NC's structural sensitivity. Starting with its shape, we consider NCs of the same number

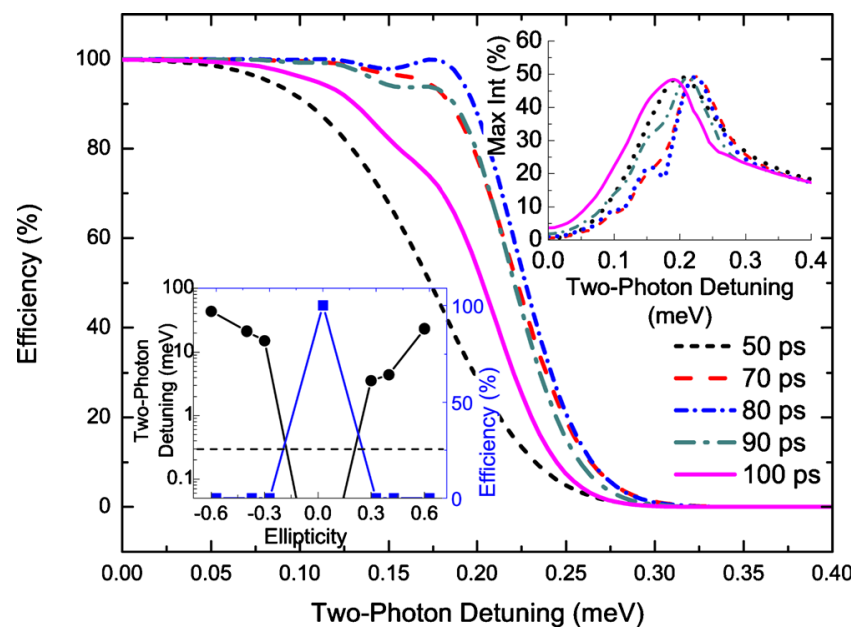

FIG. 3. (Color online) Two-photon detuning vs transfer efficiency for different time delays for the $2.1 \mathrm{~nm} \mathrm{Si} \mathrm{NC}$. Upper inset: the maximum probability of finding the electron in any of the intermediate states throughout the transfer process. Lower inset: the effect of NC ellipticity on the twophoton detuning and transfer efficiency (lines are guides to the eyes); the horizontal dashed line marks the critical $0.3 \mathrm{meV}$ two-photon detuning. of atoms (hence, same volume under zero strain) but with different asphericities as quantified by the ellipticity parameter $e$. Denoting $a$ and $b$ as the equatorial radii and $c$ as the polar radius, for oblate spheroids $(a=b>c) e=\sqrt{1-c^{2} / a^{2}}$, whereas for prolate spheroids $(a=b<c)$ we define it to be negative as $e=-\sqrt{1-a^{2} / c^{2}}$. The lower inset of Fig. 3 vividly displays the fact that the transfer is lost as the shape of the $\mathrm{NC}$ is deformed from the originally targeted geometry to which STIRAP was optimized (here spherical). The repositioning of only two NC surface atoms ( $e=0.3$ case) is enough to displace the electronic states away from the tolerable twophoton detuning window. Likewise, we observed that an incremental change in the size of the NC by including the next shell of atoms (not shown) results in a similar loss of transfer. These indicate that practically the intended STIRAP will be locked only to the single NC that it is tuned to.

In conclusion, we provide a theoretical insight for STIRAP in small Si and Ge NCs. Due to dense electronic states it displays a train of Fano resonances. The transfer can be abruptly switched off with a de voltage by introducing Stark shift that sufficiently detunes the two-photon resonance. Finally, we demonstrate the sensitivity of the transfer efficiency with respect to the structure of the $\mathrm{NC}$, which can be instrumental in addressing a single NC among an ensemble having inherent size, shape, and even local strain fluctuations.

The partial support from the European FP7 Project UNAM-Regpot Grant No. 203953 is acknowledged.

${ }^{1}$ P. Král, I. Thanopulos, and M. Shapiro, Rev. Mod. Phys. 79, 53 (2007).

${ }^{2}$ M. Saffman, T. G. Walker, and K. Mølmer, Rev. Mod. Phys. 82, 2313 (2010).

${ }^{3}$ K. Bergmann, H. Theuer, and B. W. Shore, Rev. Mod. Phys. 70, 1003 (1998).

${ }^{4}$ J. Klein, F. Beil, and T. Halfmann, Phys. Rev. Lett. 99, 113003 (2007).

${ }^{5}$ H.-H. Wang, L. Wang, X.-G. Wei, Y.-J. Li, D.-M. Du, Z.-H. Kang, Y. Jiang, and J.-Y. Gao, Appl. Phys. Lett. 92, 041107 (2008).

${ }^{6}$ A. L. Alexander, R. Lauro, A. Louchet, T. Chanelière, and J. L. Le Gouët, Phys. Rev. B 78, 144407 (2008).

${ }^{7}$ T. Takagahara, Phys. Rev. B 60, 2638 (1999).

${ }^{8}$ J. Förstner, C. Weber, J. Danckwerts, and A. Knorr, Phys. Rev. Lett. 91, 127401 (2003).

${ }^{9} \mathrm{C}$. Delerue and M. Lannoo, Nanostructures: Theory and Modelling (Springer, Berlin, 2004).

${ }^{10}$ C. Sevik and C. Bulutay, Phys. Rev. B 77, 125414 (2008).

${ }^{11}$ P. Borri, W. Langbein, S. Schneider, U. Woggon, R. L. Sellin, D. Ouyang, and D. Bimberg, Phys. Rev. Lett. 87, 157401 (2001).

${ }^{12}$ I. Sychugov, R. Juhasz, J. Valenta, and J. Linnros, Phys. Rev. Lett. 94. 087405 (2005).

${ }^{13}$ P. T. Greenland, S. A. Lynch, A. F. G. van der Meer, B. N. Murdin, C. R. Pidgeon, B. Redlich, N. Q. Vinh, and G. Aeppli, Nature (London) 465, 1057 (2010).

${ }^{14}$ C. Bulutay, Phys. Rev. B 76, 205321 (2007).

${ }^{15}$ L.-W. Wang and A. Zunger, Phys. Rev. B 59, 15806 (1999).

${ }^{16}$ C. E. Carroll and F. T. Hioe, Phys. Rev. Lett. 68, 3523 (1992).

${ }^{17}$ N. V. Vitanov and S. Stenholm, Phys. Rev. A 60, 3820 (1999).

${ }^{18}$ U. Fano, Phys. Rev. 124, 1866 (1961).

${ }^{19}$ A. E. Miroshnichenko, S. Flach, and Y. S. Kivshar, Rev. Mod. Phys. 82, 2257 (2010).

${ }^{20}$ H. Schmidt, K. L. Campman, A. C. Gossard, and A. Imamoğlu, Appl. Phys. Lett. 70, 3455 (1997).

${ }^{21}$ C. Bulutay, M. Kulakci, and R. Turan, Phys. Rev. B 81, 125333 (2010). 